\title{
Capillary Microfluidic Sensor for Determining the Most Fertile Period in Cows
}

\author{
M. Borecki ${ }^{a, *}$, M.L. Korwin-PAWlowski ${ }^{b}$, M. BebŁowska ${ }^{a}$, M. Szmidt ${ }^{c}, \mathrm{~K}_{\text {. Urbańska }}^{c}$,

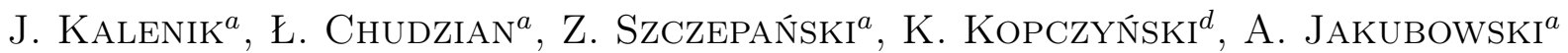 \\ AND A.J. SZMIDT ${ }^{a}$
}

${ }^{a}$ Institute of Microelectronics and Optoelectronics, Warsaw University of Technology Koszykowa 75, 00-662 Warsaw, Poland

${ }^{b}$ Département d'informatique et d'ingénierie, Université du Québec en Outaouais 101 rue Saint-Jean-Bosco, Gatineau, Québec, J8X 3X7 Canada

${ }^{c}$ Warsaw University of Life Sciences, Nowoursynowska 166, 02-787 Warsaw, Poland

${ }^{d}$ Institute of Optoelectronics, Military University of Technology, gen. S. Kaliskiego 2, 00-908 Warsaw, Poland

\begin{abstract}
This paper presents a new method of optoelectronic determination of cow fertility, based on a microfluidic fiber-optic capillary sensor. The current state of the art of fiber-optic capillary sensors is discussed briefly along with aspects of instrumentation and applications. Unlike classical fiber-optic sensors which are based on changes in light propagation inside the fiber in response to external conditions, optical capillary sensors rely on changes of light transmission within capillaries filled with the liquid to be analyzed. This approach opens up interesting new possibilities for the application of capillary microfluidic sensors, while raising specific issues relating to their construction, materials and technology. The investigated sensor uses light switching forced by local heating in a capillary filled with bovine vaginal fluid. The dynamically recorded data were processed in an artificial neural network. We were able to observe changes in the cow's fertility cycle allowing us to identify when an individual cow was in the middle part of standing estrus which is most situated for insemination.
\end{abstract}

PACS numbers: 42.81.Pa, 87.85.fk, 47.35.Pq, 07.05.Mh

\section{Introduction}

Infertility has been identified as the number one problem on dairy farms [1]. In "An International Conference: Fertility in Dairy Cows - Bridging the Gaps", the thesis that "applied research must retain a clear vision of how research findings can be translated into changes and improvements in fertility performance at the farm level" was postulated [2].

The association between milk urea nitrogen (MUN) and the fertility of dairy cows was studied by Rajala-Schultz et al. using field data [3]. Cows with MUN levels below 10.0 were 2.4 times more likely and cows with MUN levels between 10.0 and $12.7 \mathrm{mg} / \mathrm{dl}$ were 1.4 times more likely to be confirmed pregnant than cows with MUN values above $15.4 \mathrm{mg} / \mathrm{dl}$. The results indicated that increasing MUN levels appeared to be negatively related to dairy cow fertility and were associated with a lower likelihood of detectable pregnancy at herd checks. They also suggested that MUN levels adversely associated with fertility might be lower than had been reported earlier.

Milk urea testing as a tool to monitor reproductive performance was evaluated by Godden et al. [4]. When

* corresponding author; e-mail: borecki@imio.pw.edu.pl interpreted at the individual cow level, there was no association between milk urea and the likelihood of pregnancy from an insemination occurring within the 45-day period preceding test day. However, it was found that while milk urea measurements might be useful as a management tool to improve the efficiency of production or reduce nitrogen excretion, by helping to optimize the efficiency of protein utilization, they had limited utility as a monitoring or diagnostic tool in the area of reproductive performance.

The technology of fertility sensing established for humans relies on continuous sensing and recording of the body temperature, analyzing the urinary luteinizing hormone level, examining the chloride concentration in saliva or observing the vaginal discharge [5-7]. In contrast, there are no technologies available for on-farm testing to predict the fertility of cows. Moreover, existing laboratory-based sensing devices are not exact and are affected by external conditions such as outdoor temperature, salt consumption by the cows and humidity changes, resulting in a poor record of fertility prediction.

The similarity of vaginal fluid between different species of mammals can be used to advantage for the investigation of cow fertility sensors. However, the idea of simply using an optical microscope combined with a sensitive procedure is not at all appropriate when it comes to as- 
sessing cows in the diary barn or outdoors on the farm. The objective of our work was therefore to develop a new sensor and a new method for predicting the most fertile phase of the estrus cycle of cows.

\subsection{The vaginal fluid of mammals}

The vaginal secretions of all mammals include pyridine, squalene, urea, acetic acid, lactic acid, complex alcohols (including cholesterol), glycols (including propylene glycol), ketones and aldehydes [8]. The vaginal fluid is slightly acidic and may become more acidic with certain sexually transmitted diseases. The normal $\mathrm{pH}$ of vaginal fluid is between 3.8 and 4.5 [9]. The fluid is typically clear. It varies in consistency, texture and color, depending on the phase of the estrus cycle, the presence of infection, certain drugs (legal or illegal), genetic factors and diet. The vagina's aliphatic acids are generated by the metabolic processes of resident bacteria, including Lactobacilli, Streptococci, and Staphylococci. For all mammals, the acid content varies with the estrus cycle, rising from the first day of the cycle and peaking mid-cycle, just before ovulation. Vaginal fluid may be present only at ovulation, a few days before ovulation, or throughout the entire cycle. When vaginal fluid is present at times other than the ovulation phase, it is turbid and its volume tends to be very small. For example, the volume of vaginal liquid of a cow may be in the order of $50 \mathrm{ml}$. The vaginal fluid from the ovulation or fertile phase is often described as transparent, glassy, ductile and viscid. The term "viscid" can be easily misunderstood. It refers in fact to a quality of stickiness that is more connected with surface tension of the vaginal fluid than with its viscosity or tendency to not flow freely. The biological role of vaginal fluid in mammals is to enable the transfer of semen through the vagina to the uterus in the fertile phase and to block the way for contaminants during other phases of the estrus cycle.

\subsection{The most fertile phase of cows from the biological point of view - standing estrus and ovulation}

Cows are most fertile when in standing estrus, also referred to as standing heat, or standing to be mounted (STBM). This is also the period when a cow is sexually receptive. A cow enters standing estrus gradually. Prior to standing estrus she may appear nervous and restless, for example, walking a fence line in search of a bull or bawling more than usual. Other signs that a cow might be in standing estrus are a roughed-up tail, a clear mucous discharge from the vagina, and a swollen vulva. However, the only conclusive sign that a cow is in estrus is that she stands to be mounted by other cattle. Prior to STBM she will usually try to mount other animals. These signs will be present until standing estrus occurs. Following standing estrus, an ovarian follicle will release the egg it contains. This rupture of the follicle is referred to as ovulation and occurs between 24 and $32 \mathrm{~h}$ after the onset of standing estrus. Following its release, the egg will enter the female reproductive tract and be fertilized if the female has been mated [10]. Following each standing estrus, a new estrus cycle will be initiated. In a normally cycling animal the interval between each standing estrus should be about 21 days, but the range in estrus cycle length is from 17 to 24 days [11].

Estrus in cattle on average lasts about $15 \mathrm{~h}$, ranging from less than $6 \mathrm{~h}$ to close to $24 \mathrm{~h}$. The percentage of STBM cows has declined from $80 \%$ to $50 \%$ over the past 30-50 years and the duration of STBM has decreased from $15 \mathrm{~h}$ to $5 \mathrm{~h}$. These changes have occurred in parallel with a reduction in first-service-pregnancy rate from $70 \%$ to $40 \%[12,13]$. Clearly, there is a strong need now for a sensor that can predict the STBM phase with some precision.

\subsection{Fiber-optic capillary sensing methods}

Unlike classical fiber-optic sensors which rely on changes in light propagation inside the fiber as affected by outside conditions, optical capillary sensors rely on changes of light transmission in capillaries filled with the liquid to be analyzed [14]. They open interesting new possibilities for the applications of sensors, while raising specific issues relating to their construction, materials and technology which are presented in greater depth in the existing literature $[15,16]$.

Optical capillary sensor heads can be in the form of a fiber-optic capillary (FOCap) or a liquid core waveguide (LCW) as illustrated in Fig. 1. The sensor heads

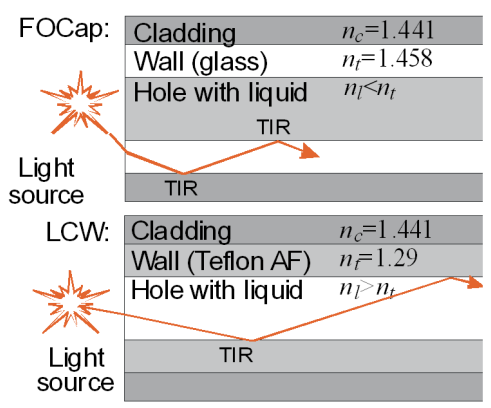

Fig. 1. The light propagating in optical capillary heads: FOCap - fiber-optic capillary, LCW — liquid core waveguide.

are often designed as fixed components in microfluidic systems, but disposable heads are required for the examination of organic fluids. The replaceable capillary heads are equipped with one or more photoluminescence sensor layers when they are to sense the presence of different ions in the examined fluid [17]. The advantages of replaceable capillary heads in biological fluid examinations are the very small sample volume that is required for analysis and the fact that there is no need to wash out the capillaries. For best accuracy the examination has to be multi-parametric.

The new method for sensing multiple parameters of liquids using a fixed capillary head proposed in [18] is based on light switching of ballistic rays that propagate 


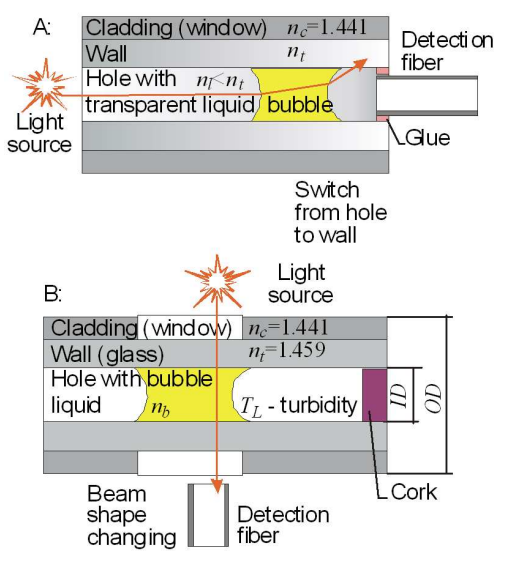

Fig. 2. Light propagation in optical capillary heads using different sensing methods: A - switching from hole to wall, B - beam shape changing.

in a transparent liquid with an index of refraction $n_{l}$ that is lower than the refractive index of the capillary wall $n_{t}$ as presented in Fig. 2A. Glue is used to hold the detection fiber securely in place and to keep steady the position of the liquid. A bubble is created by local heating of the capillary. The signal is present in the detection fiber when there is no bubble present in the capillary.

The proposed new method of multi-parametric liquid sensing utilizes beam shape changing as presented in Fig. 2B. It is suitable for implementation as a disposable capillary optrode. Moreover, the turbidity of the fluid is not a problem here, which is important when analyzing the vaginal fluid. The capillary is partially filled with liquid and cork is inserted at the end of the capillary that is in contact with the liquid. This enables the creation of a bubble in the capillary with a corresponding dislocation of the liquid. For transparent and semitransparent liquids, light intensity is greater in the detection fiber when there is no bubble than when a bubble is present.

\section{Principles of sensor construction and operation}

\subsection{Arrangement of the capillary}

The operation of our optical capillary sensor is based on biological changes in the vaginal fluid of mammals affecting its transparency. For sensing purposes, the capillaries must first be filled. This can be achieved through the capillary effect of a liquid wetting the walls of the capillary by surface tension. In most biological applications, where the liquid content is mainly water, the capillary can be filled without a pump when the inner diameter (ID) of the capillary is small enough. On the other hand, a larger ID makes for easier coupling of light. In our experiments, we relied on capillary action to fill $60 \mathrm{~mm}$ lengths of capillaries from two different suppliers, Polymicro Technologies Inc. of Phoenix, Arizona and the Department of Optical Radiation of Białystok University of Technology $[19,20]$, both with $\mathrm{ID}=700 \mu \mathrm{m}$, achieving heights as shown in Table.

TABLE Capillary action height (cah) for capillary with ID = $700 \mu \mathrm{m}$.

\begin{tabular}{l|c}
\hline \hline Fluid type & cah [mm] \\
\hline water & 28 \\
vaginal fluid for cow in middle of STBM & 20 \\
vaginal fluid for cow in pregnant phase & 0 \\
vaginal fluid for cow with suppurative mat- & 40 \\
ter/pyometra &
\end{tabular}

The length of time required to fill the capillary depends on the kind of fluid. The vaginal fluid of a cow in the middle of STMB fills the capillary in $2 \mathrm{~s}$, while fluid collected $6 \mathrm{~h}$ after the middle of STMB fills capillary in 3 to $6 \mathrm{~s}$. Centrifuging of the sample increases the capillary filling speed by a factor of 2 to 3 . Once the capillary has been filled, the fluid does not flow out, but merely forms a drop at the end of it. We cleaned off the drop by swabbing the capillary after positioning it at $10^{\circ}$ to the horizontal. The position of the fluid in the capillary was fixed by closing one end of the capillary with a plasticine stopper about $3 \mathrm{~mm}$ long. Unlike glues, the plasticine does not wet the capillary, nor does it react with the vaginal fluid.

\subsection{Optical configuration of the sensor}

The power distribution of the optical signals has to be considered in the configuration of the sensor. We investigated the reliability of sensing with a replaceable capillary in the sensor head shown in Fig. 3. V-grooves

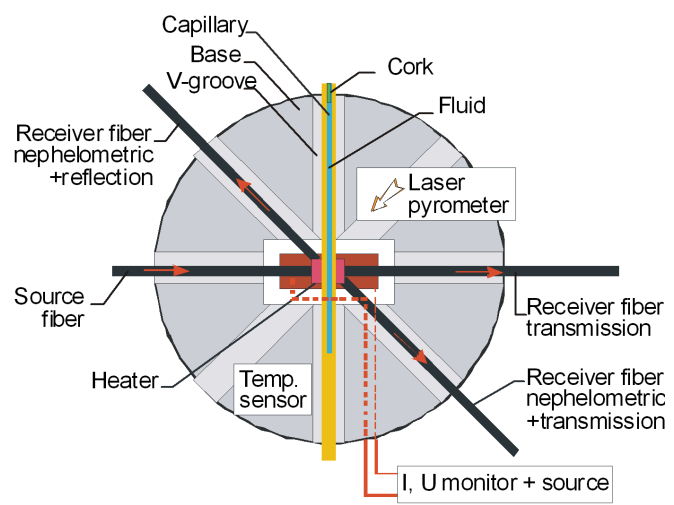

Fig. 3. Configuration of the sensor head used for experiments.

were made to position the capillaries intersecting at $45^{\circ}$ degrees at the center of the base of the sensor head.

We used two types of large-core silica glass optical fiber, FVA8008801100 and FVA100010501250, from Polymicro Technologies Inc. The optical fibers were 
mounted on the head with magnetic clamps, with micromechanical pressure pads or with glue and were terminated on the free end with SMA connectors. The capillary was mounted on the base with magnetic clamps that can hold it securely at up to $10 \mathrm{~g}$ of acceleration.

Two different configurations were used. The first configuration worked without light modulation and contained commercially available instruments: Fiber-Coupled Laser Source S1FC675 (ThorLabs) and Dual-Channel Optical Power Meter PM 300 (ThorLabs) with two S120B detectors. For light coupling to the source fiber, we used additional multimode patch cables with a core diameter of $62.5 \mu \mathrm{m}$, terminated with FC/PC connectors and FC/SMA adapters. The second configuration worked with light modulation, was made in-house and consisted of a LED light source and two single-channel optoelectronic interfaces, and was limited to two receiver channels.

The laser source allows the delivery of $2 \mathrm{~mW}$ of optical power into the source fiber, while the LED source can deliver $100 \mu \mathrm{W}$. The modulation of the light source, filtering and envelope demodulation in an optoelectronic interface compensates for the difference in power and makes the sensor insensitive to ambient light. The signals from the PM 300 and the optoelectronic interfaces were fed to an IoTech PersonalDaq/3001 data acquisition card and were processed in the DASYLab programming environment that also controlled the heater.

The signal intensity is strongest in the transmission arm and is sensitive to the presence of transparent and semitransparent fluids such as vaginal fluid, and to turbid fluids such as milk. The information contained in the formation of a bubble depends on the turbidity of the liquid and can be extracted by measuring the signal in the two nephelometric arms. The relative change in the optical signal in the presence of milk, water or transparent vaginal fluid is two orders of magnitude greater in the nephelometric arms than in the transmission arm. The signals in nephelometric and reflection arms depend strongly on the position of the capillary.

\subsection{Design and fabrication of the sensor}

To make a low-cost, reliable and universal configuration of sensor head with a replaceable capillary optrode, the designer has to consider the mechanical stabilization of the optical elements and the power balances of heat transfers. The capillaries have to be positioned just above the heater taking into account the tolerances of the capillary diameters of $\pm 20 \mu \mathrm{m}$. Micromechanical, hybrid and monolithic technologies can be employed in fabricating the sensor head. For stable positioning of the replaceable capillary one can use magnetic clamps.

The micromechanical capillary head was made of magnetic tool steel. It has a cylindrical shape with a diameter of $5 \mathrm{~cm}$ and a thickness of $4 \mathrm{~mm}$ with $\mathrm{V}$-grooves machined for the optical fibers, threads for screws and slots for the heater and wire leads, as illustrated in Fig. 4.

The critical point of the described construction is its reliable three-dimensional positioning of the heater which

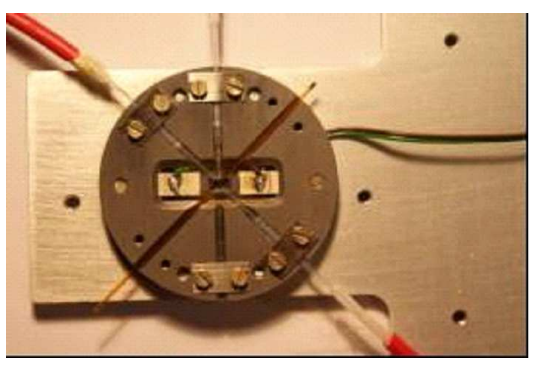

Fig. 4. View of capillary head base with mounted fibers, heater and a capillary in position — without magnetic clamps.

was made using thick film technology and shaped by laser.

Silicon hybrid and monolithic techniques were used in the next phase of this work to integrate the heater and the base. The silicon ( $\mathrm{Si}$ ) which replaced steel as the base material must be thick enough to allow the planar placement of the fibers and capillary and their positioning over the heater. To accommodate the fibers and replaceable capillaries with an outer diameter of $850 \mu \mathrm{m}$ we used a base with a thickness of $800 \mu \mathrm{m}$ and side length of $30 \mathrm{~mm}$, on which the $\mathrm{V}$-grooves and the heater with leads were made as presented in Fig. 5.

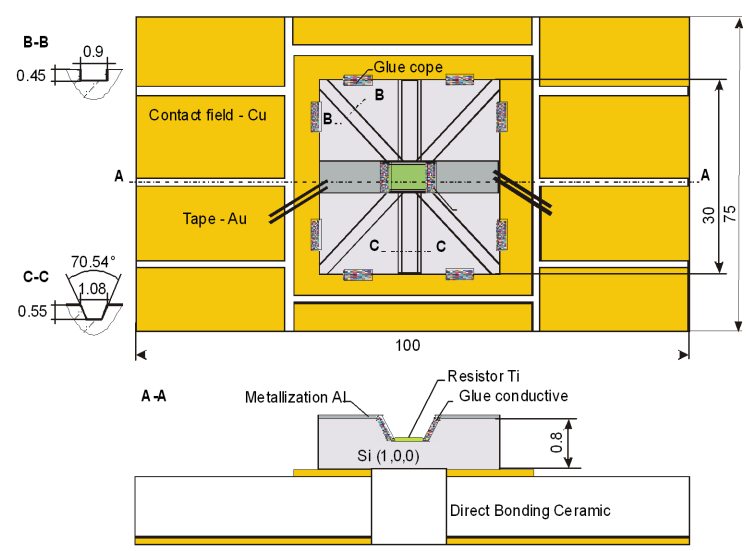

Fig. 5. Construction of the integrated sensor head base.

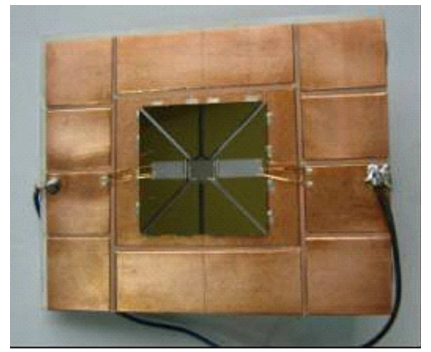

Fig. 6. View of integrated sensor head base. 
The tapered V-grooves were etched in the $\langle 100\rangle$ orientation silicon using anisotropic etch. The V-shape allows positioning of the replaceable capillary $100 \mu \mathrm{m}$ over the heater. The etching process was used also to form the heater area but could not be used with planar fiber configuration. In that case the grooves were sawed in the silicon with a diamond blade. Next, the heater resistor and leads were sputtered and finally the conductive glue was deposited on the sloping areas of the resistor. The Si structure was mounted with glue on direct bonding ceramics (DBC). The hole underneath the resistor area which minimizes heat transfer from the resistor to the DBC was drilled with a laser. The integrated sensor head base is shown in Fig. 6. The fibers in this structure were mounted with glue. The capillary was positioned with magnetic clamps while the structure was placed on a permanent magnet.

The temperature of the heaters was monitored directly by a laser pyrometer and indirectly by adjusting the current of the heater resistor. The temperature in the capillary was monitored by observing the boiling point of the various fluids filling the capillary. With the capillary positioned $100 \mu \mathrm{m}$ above the heater, the heater temperature had to be $180^{\circ} \mathrm{C}$ for water to boil. A temperature of $100^{\circ} \mathrm{C}$ in the capillary liquid was reached for the micromechanical capillary base with a heater power of $3 \mathrm{~W}$, and for the integrated sensor head base with a heater power of $10 \mathrm{~W}$. Both types of base structure have their advantages. The micromechanical base is easily reconfigurable and has lower power consumption, while the integrated structure is more suitable for mass production.

\section{Experimental results}

\subsection{Experimental sample material}

In our experiments, we used vaginal fluid collected from cows at a farm while the inseminator was determining the estrus phase of individual cows by the exact location, size and firmness of the cervix. The samples were collected in the middle of the STBM phase and $6 \mathrm{~h}$ later. The middle of STMB was individually observed for each cow.

A Foley-type catheter was introduced into the cow's cervix during estrus and left in place for $3 \mathrm{~min}$. The secretion was drawn and then transferred to sterile tubes by delicate movements of the catheter. The tubes were transported in frozen state to the laboratory where they were examined using the developed sensor after being thawed. Some samples were examined directly and some were centrifuged before examination.

\subsection{Analysis of the sensor signal}

The signals received from the transmission and the nephelometric arms of the micromechanical head were examined after sample heating with $2 \mathrm{~W}$ and $3 \mathrm{~W}$ power. The heating automatically stops after the transmission signal speed of change drops below 1 arb.u. $/ 0.1 \mathrm{~s}$ or at the end of $60 \mathrm{~s}$ of the measurement cycle, whichever comes

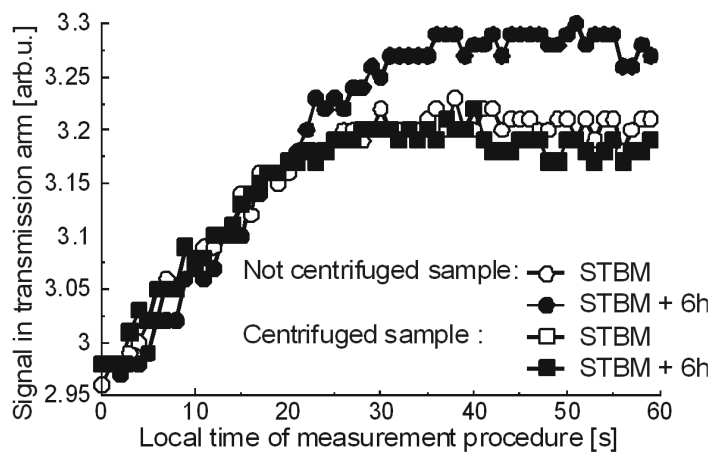

Fig. 7. Signal in the transmission arm during heating of the vaginal fluid with $2 \mathrm{~W}$ power.

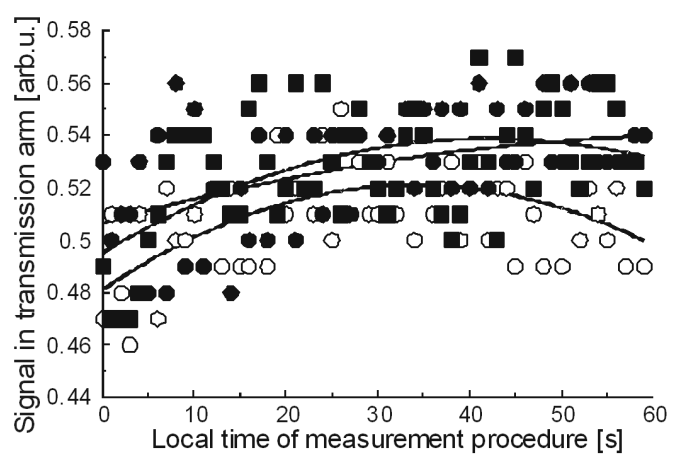

Fig. 8. Signal in nephelometric and reflection arm during heating of vaginal fluid with $2 \mathrm{~W}$ power. Symbols as in Fig. 7.

first. The signals collected for samples heated with a power of $2 \mathrm{~W}$ are presented in Figs. 7 and 8.

The signal axis in Fig. 8 has a range of 0.14 arb.u. (from 0.44 up to 0.58 arb.u.) in contrast to the signal range of 0.35 arb.u. shown in Fig. 7, so that the point spreads for any signal trace on both figures are the same. The signal values and the speed of their changes measured in the transmission arm of the sensor are greater than those measured in the nephelometric and reflection arms. It seems that the heating power of $2 \mathrm{~W}$ is not sufficient for bubble creation in the vaginal fluid and therefore inadequate for maximum fertility phase identification, but the signal changes observed during the measurement procedure indicate that some less-than-obvious changes nonetheless occur in the fluid [21, 22]. When the heater power was increased to $3 \mathrm{~W}$, a bubble was created in the fluid. The bubble formation is manifested in the rapid transmission signal changes presented in Figs. 9 and 10, where results from centrifuged samples are given.

The bubble creation time differs for samples collected in the middle of the fertile STMB phase and samples collected $6 \mathrm{~h}$ later. The bubbles created in the STBM samples do not disappear rapidly after the heating is off. This can be utilized to recognize the fertility state of a cow using artificial neural network (ANN) analysis. A basic multilayer perceptron can be used with the following 


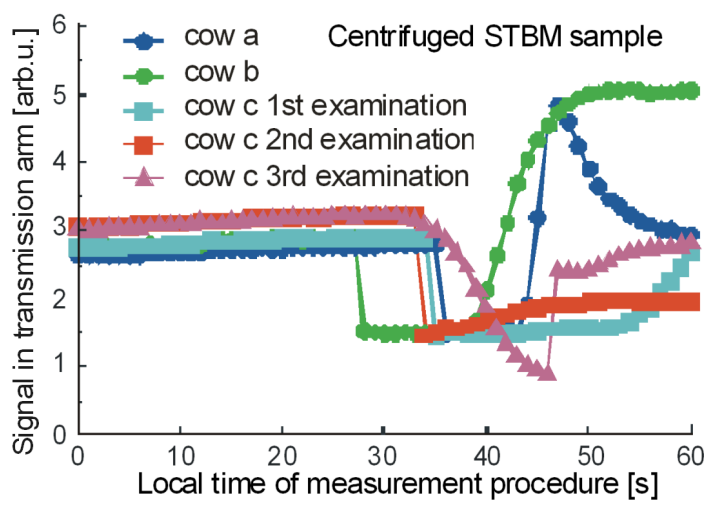

Fig. 9. Signal in transmission arm during heating of vaginal fluid with $3 \mathrm{~W}$ power for samples collected in the middle of the STBM phase of cow fertility.

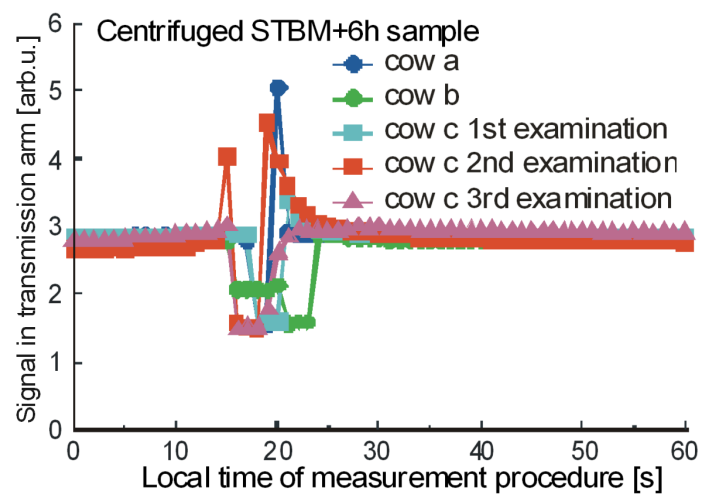

Fig. 10. Signal in transmission arm during heating of vaginal fluid with $3 \mathrm{~W}$ power for samples collected $6 \mathrm{~h}$ after the middle of the STBM phase of cow fertility.

configuration: 3 inputs, 1 output, 3 layers and the sigmoid transfer function $[23,24]$. For the data model we selected the initial signal level of the time of vapor bubble creation, and the time when the signal level stabilized at the initial level. We used the Qnet software for our ANN analysis. The correlation of the input and output factors given by Qnet after ANN learning was 0.998, which allows us to state that the most fertile time in a cow's estrus cycle can be reliably predicted with our method.

\section{Conclusion}

Disposable capillary microfluidic sensors can be used to monitor cow fertility in order to establish the best time for breeding or insemination. The proposed method of fertility classification of cows is based on examining the vaginal fluid of the cow. This fluid enables semen transfer through the vagina to the uterus in the fertile phase and blocks contamination in the non-fertile phase. Our method offers improved efficiency and economy by eliminating microfluidic pumps as well as eliminating the need for hormonal tests. A further advantage is that the proposed novel optoelectronic method of vaginal fluid ex- amination requires only a low sample volume, thereby allowing monitoring over a longer period, even a cow's entire estrus cycle. A single examination requires a mere $15 \mathrm{ml}$ of fluid volume and $60 \mathrm{~s}$ of measurement procedure. The $6 \mathrm{~h}$ resolution of determination of cow maximum fertility phase achieved in the tests of the proposed method is very satisfactory.

\section{Acknowledgments}

This work was supported in part by grants from the Discovery Program of the Natural Sciences and Engineering Research Council of Canada and from the Canada Foundation for Innovation, and by the European Union structural funds grant MNS-DIAG task $2 \mathrm{~A}$.

The micromechanical version of the capillary base was fabricated in cooperation with Communication Equipment Factory II — State Aviation Works of Poland (WSK II - PZL Warszawa). The silicon structure of the MEMS-hybrid capillary base was fabricated in cooperation with Institute of Electron Technology in Warsaw.

\section{References}

[1] http://www.ruralni.gov.uk/index/livestock/ livestock_dairy/breeding/fertility.htm .

[2] http://www.bsas.org.uk/downloads/ Fertility_all_files.pdf .

[3] P.J. Rajala-Schultz, W.J.A. Saville, G.S. Frazer, T.E. Wittum, J. Diary Sci. 84, 482 (2001).

[4] S.M. Godden, D.F. Kelton, K.D. Lissemore, J.S. Walton, K.E. Leslie, J.H. Lumsden, J. Dairy Sci. 84, 1397 (2001).

[5] J. Lennard, J. Lind, M. Honeywell, http: //www. ovwatch.com/downloads/ USPharmacist.pdf .

[6] R.J. Fehring, W.D. Schlaff, J. Nurse-Midwifery 43, 117 (1998).

[7] L. Ngalamou, D. Rose, in: Proc. 15th IEEE Symp. on Computer-Based Medical Systems (CBMS'02), Eds. M. Sonka, C. Kuiko, University of Maribor, Maribor (Slovenia) 2002, p. 335. http://doi.ieeecomputersociety.org/10.1109/ CBMS . 2002.1011400.

[8] P. Spinrad, Guide to Body Fluids, The RE/Search, San Francisco 1994.

[9] N.A. Ryan-Wenger, J.L. Neal, A.S. Jones, N.K. Lowe, Nurs Res. 59, 2 (2010).

[10] B. Mcguirk, In Practice 26, 272 (2004).

[11] E. Mukasa-Mugerwa, A Review of Reproductive Performance of Female Bos Indicus (Zebu) Cattle, ILCA Monograph 6. ILCA (International Livestock Centre for Africa), Addis Ababa, Ethiopia 1989.

[12] H. Dobson, S.L. Walker, M.J. Morris, J.E. Routly, R.F. Smith, in: An Int. Conf.: Fertility in Dairy Cows - Bridging the Gaps, Ed. M. Rayen, University of Liverpool, Liverpool (UK) 2007, p. 27.

[13] P.M. VanRaden, A.H. Sanders, M.E. Tooker, R.H. Miller, H.D. Norman, M.T. Kuhn, G.R. Wiggans, J. Dairy Sci. 87, 2285 (2004). 
[14] B.K. Keller, M.D. DeGrandpre, C.P. Palmer, Sensors Actuators B 125, 360 (2007).

[15] M.E. Lippitsch, S. Draxler, D. Kieslinger, H. Lehmann, B.H. Weigl, Appl. Opt. 35, 3426 (1996).

[16] M. Borecki, M.L. Korwin-Pawlowski, M. Beblowska, IEEE Sensors J. 8, 1208 (2008).

[17] D. Kieslinger, S. Draxler, K. Trznadel, M.E. Lippitsch, Sensors Actuators B 38-39, 300 (1997).

[18] M. Borecki, M.L. Korwin-Pawlowski, P. Wrzosek, J. Szmidt, Meas. Sci. Technol. 19, 065202 (2008).

[19] R. Romaniuk, J. Dorosz, Proc. SPIE 6347, 634710 (2006).
[20] D. Dorosz, K. Barczak, T. Pustelny, J. Dorosz, Acta Phys. Pol. A 114, A-61 (2008).

[21] K. Gut, D. Nabaglo, Acta Phys. Pol. A 116, 307 (2009).

[22] P. Kaniewski, J. Kozubek, Acta Phys. Pol. A 116 , 325 (2009).

[23] M. Borecki, Sensors 7, 384 (2007).

[24] M. Borecki, M. Szmidt, M.L. Korwin-Pawlowski, M. Beblowska, T. Niemiec, P. Wrzosek, Photon. Let. Pol. 1, 37 (2009). 